\title{
A LOCALIZED RECURSIVE ESTIMATION ALGORITHM FOR VECTOR PARAMETER ESTIMATION IN AD HOC WIRELESS SENSOR NETWORKS
}

\author{
Amir Rastegarnia - Mohammad Ali Tinati - \\ Behzad Mozaffari — Azam Khalili
}

\begin{abstract}
In this paper a localized recursive estimation scheme for vector parameter estimation in ad hoc wireless sensor networks (WSNs) is proposed. In our setup, each sensor has a noisy measurement from a fixed, but unknown vector parameter. The classical best linear unbiased estimator (BLUE) cannot be implemented in a practical ad hoc sensor network due to its requirement to transmit all real-valued messages to a fusion center (FC). To address this problem, in this paper we propose a recursive estimation algorithm which is based on progressive cooperation between sensors. In our proposed algorithm, each sensor computes an estimate of the unknown parameter using its own local data and some information from prior sensor. The computed estimate is delivered to the next sensor and so on. The last sensor delivers the computed estimate of the unknown parameter to the destination. Our mathematical analysis shows that the proposed recursive algorithm is an unbiased estimator of the unknown parameter and asymptotically approaches to the estimate that would be obtained if each sensor had access to the information across the entire network. Also, the simulation examples are used to show the performance of the proposed scheme.
\end{abstract}

K e y w or ds: ad hoc network, distributed estimation, wireless sensor network, BLUE, estimation

\section{INTRODUCTION}

A wireless sensor network consists of a large number of sensors, where each sensor is capable of sensing, processing and transmitting environmental information. These capabilities make it possible to use WSNs in many applications such as environmental monitoring, space exploration and target localization [1]. In most of these applications one need to estimate an unknown parameter using discrete-time samples collected across sensors. Due to implementation considerations, it is not possible to send all data samples to a central location for processing. To address this problem distributed estimation schemes have been introduced in the literature. Based on WSN topology, ie WSN with FC and ad hoc WSN, there are two different ways for distributed estimation [2]. The main goal in distributed estimation algorithms is to distribute the computations of estimation task between sensors and FC (in FC based WSN) and totally between sensors in ad hoc WSNs.

\subsection{Related Works}

In [3-7] some distributed estimation algorithms in WSNs have been proposed. In a FC-based distributed estimation, the basic idea is to reduce the bits needed to encode the local measurements. The generated bits in sensors are sent to FC where an estimate is calculated by using appropriate processing of received messages. In [3], distributed estimators for known univariate noise pdfs (probability density function), and known noise pdfs with a finite number of unknown parameters have been proposed. In [4] the same problem for unknown noise pdf is considered. As shown in [3] and [4], optimal distributed estimators depend on the parametric model and the noise pdfs. In certain cases, characterizing the exact sensor observation distributions for a large number of sensors may be impossible, especially in a dynamic sensing environment. Such applications motivate universal distributed estimators that are independent of the noise or parameter distributions, under either bandwidth or energy constraints [2].

In [5] and [6], universal distributed estimation algorithms with one-bit message encoding functions have been proposed for homogeneous and inhomogeneous environments respectively. In an inhomogeneous sensing environment measurement of different sensors have not the same quality. To consider the quality of measurements, in [6] the length of an encoded message is decided by the local signal to noise ratio (SNR). In the given algorithms in [3-6] there is no any cooperation between sensors, but as shown in [7], the performance of estimator can be improved if each individual sensor dynamically adjusts the threshold of its quantizer based on earlier transmissions from other sensors.

When the distance between FC and sensors is long and/or multi-hop communications have to be used, the transmission of these messages not only consumes much energy but also increases network traffic load even if the

* Faculty of Electrical and Computer Engineering, University of Tabriz, Tabriz 51664, Iran, \{rastegar, tinati, mozaffary, a-khalili\} @tabrizu.ac.ir 


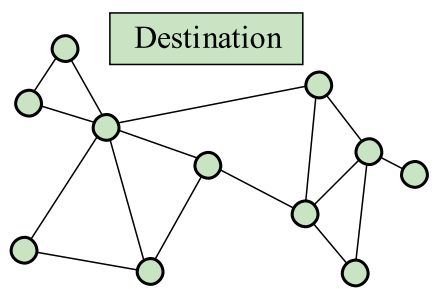

Fig. 1. An ad hoc wireless sensor network with $N$ sensors

messages are short [8]. Furthermore, it is generally believed that in a WSN the energy required for local computation is much less than that used for communications [9]. These limitations of FC based distributed estimation are not encountered with ad hoc WSNs where each sensor communicates only with its neighbors, and the estimation task can be performed in a totally distributed fashion [10]. Recently distributed adaptive estimation algorithms [11-17] have been proposed for distributed estimation in ad hoc sensor networks. In [11-13] distributed adaptive estimation algorithms using incremental optimization techniques are developed and their transient and steady-state performance analyses are also provided. IDLMS (incremental distributed least mean square) [11] and distributed recursive least mean-square (DRLS) [12] are the examples of such algorithms. These algorithms are distributed, cooperative, and able to respond in real time to changes in the environment.

In [14-17], diffusion implementations of distributed adaptive estimation algorithms are developed. In these algorithms, each node can communicate with all its neighbors as dictated by the network topology. Both LMSbased and RLS-based diffusion algorithms have been considered in the literature [14], [15]. In addition, for both of these algorithms the performance analysis can be found in [16] and [17] respectively. In comparison with incremental based algorithms, diffusion based methods need more communication resources while have better estimation performance.

In [18] a recursive algorithm for scalar parameter estimation in ad hoc wireless sensor networks is considered. In this paper we extend the work in [18] and propose a localized recursive estimation algorithm for vector parameter estimation in ad hoc wireless sensor networks. In our setup, it is assumed that the computed estimate should deliver to a destination. The proposed scheme performs the estimation locally and recursively by the sensors measuring the parameter. Each sensor performs estimation based on its own measurement and the intermediate estimation results from its upstream sensor. The last sensor delivers the computed estimate to the destination. Unlike incremental distributed adaptive estimation algorithms, in our proposed method it is not necessary to have a cycle between sensors. Our mathematical analysis shows that the proposed method is an unbiased estimator and asymptotically approaches to the estimate that can be achieved by a FC with all real-valued measurements.

\subsection{Paper Structure and Notation}

The rest of the paper is organized as follows. In section 2 we present the problem formulation for parameter estimation in sensor networks. A recursive parameter estimation scheme is provided and its properties are analyzed in Section 3. Section 4 gives some numerical simulation results and concluding remarks are discussed in Section 5 . Through this paper, we will use lower case letters (like $a$ ) for scalars, bold lower case letters for vectors (like a) and upper case letters (like $A$ ) for matrices. The scalar, vector and matrix at iteration $k$ are represented by $a_{k}, \mathbf{a}_{k}$ and $A(k)$ respectively. In addition ()$^{\top}$ is used for matrix transpose and $E$ stands for statistical expectation.

\section{PROBLEM STATEMENT}

Consider an ad hoc wireless sensor network with $N$ sensors as shown in Fig. 1. Each sensor's observation is modeled as

$$
x_{k}=\mathbf{u}_{k} \theta+v_{k}, \quad k=1,2, \ldots, N
$$

where $\theta \in \mathbb{R}^{M \times 1}$ is a fixed (but unknown) vector parameter, $v_{k}$ is the observation noise and

$\boldsymbol{u}_{k}=\left(u_{k, 1}, u_{k, 2}, \ldots, u_{k, M}\right)$ is the row regressors vector (a known vector) for the $k$ th sensor. We collect $x_{k}, v_{k}$ and $u_{k, i}$ to form the following vectors and matrix

$$
\begin{gathered}
\mathbf{x}=\left[\begin{array}{c}
x_{1} \\
x_{2} \\
\vdots \\
x_{N}
\end{array}\right]_{N \times 1}, \quad \boldsymbol{v}=\left[\begin{array}{c}
v_{1} \\
v_{2} \\
\vdots \\
v_{N}
\end{array}\right]_{N \times 1} . \\
U=\left[\begin{array}{c}
\mathbf{u}_{1} \\
\mathbf{u}_{2} \\
\vdots \\
\mathbf{u}_{N}
\end{array}\right]_{N \times M}=\left[\begin{array}{cccc}
u_{1,1} & u_{1,2} & \ldots & u_{1, M} \\
u_{2,1} & u_{2,2} & \ldots & u_{2, M} \\
\vdots & \vdots & \ddots & \vdots \\
u_{N, 1} & u_{N, 2} & \ldots & u_{N, M}
\end{array}\right] .
\end{gathered}
$$

The observation noises are assumed to be spatially uncorrelated white noise with zero mean and variance $\sigma_{k}^{2}$. The covariance matrix of noises is given by

$$
R=\mathrm{E}\left\{\boldsymbol{v} \boldsymbol{v}^{\top}\right\}=\left[\begin{array}{cccc}
\sigma_{1}^{2} & 0 & \cdots & 0 \\
0 & \sigma_{2}^{2} & 0 & 0 \\
\vdots & \vdots & \ddots & \vdots \\
0 & 0 & \cdots & \sigma_{N}^{2}
\end{array}\right]
$$

Note that $R$ is symmetric and positive definite matrix. A well known best linear unbiased estimator (BLUE) [19] can be applied to estimate $\theta$ and to achieve a minimum MSE. According to BLUE, when measurements are available, the estimator $\hat{\theta}$ of the parameter $\theta$ is given by [19]

$$
\hat{\theta}=\left(U^{\top} R^{-1} U\right)^{-1} U^{\top} R^{-1} \mathbf{x}
$$




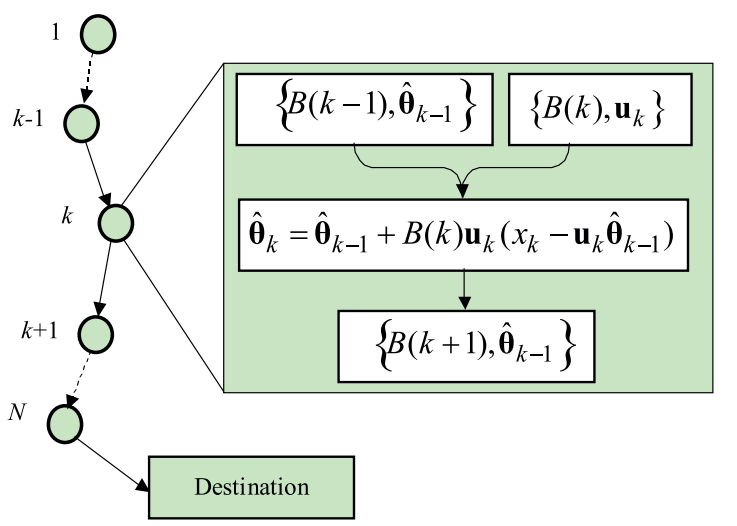

Fig. 2. Illustrative scheme of our localized recursive estimation algorithm

The MSE of BLUE is given as

$$
\mathrm{E}\left[(\theta-\hat{\theta})^{2}\right]=\left(U^{\top} R^{-1} U\right)^{-1}
$$

To use (4), it is necessary that $\boldsymbol{x}$ and $U$ be available in the FC which in turn needs a lot of communications and computation recourses. To address this problem, we propose a recursive BLUE for the above estimation problem.

\section{PROPOSED ALGORITHM}

To develop our recursive algorithm, without lose of generality, we assume that $\sigma_{k}^{2}=1, \forall k$. By this assumption, we have $R=I$. It is straightforward to extend the derived formulas for the $\sigma_{k}^{2} \neq \sigma_{j}^{2}, j \neq k$ case. The following proposition provides a recursive BLUE for the mentioned estimation problem

Proposition 1. In the $k$ th sensor $\theta$ is estimated by the following recursive equation

$$
\hat{\theta}_{k}=\hat{\theta}_{k-1}+B(k) \mathbf{u}_{k}^{\top}\left(x_{k}-\mathbf{u}_{k} \hat{\theta}_{k-1}\right), \quad \hat{\theta}_{0}=\boldsymbol{O}
$$

where $B(k)$ is defined as

$$
B(k) \triangleq\left(U_{k}^{\top} R_{k}^{-1} U_{k}\right)^{-1}=\left(U_{k}^{\top} U_{k}\right)^{-1}
$$

and is updated via

$$
B^{-1}(k)=B^{-1}(k-1)+\mathbf{u}_{k}^{\top} \mathbf{u}_{k}, \quad B^{-1}(0)=\varepsilon I,
$$

In (7) $\varepsilon$ is small positive constant. We further use the following notations

$$
\mathbf{x}_{k}=\left[\begin{array}{c}
x_{1} \\
x_{2} \\
\vdots \\
x_{k}
\end{array}\right]_{k \times 1}, \quad U_{k}=\left[\begin{array}{c}
\mathbf{u}_{1} \\
\mathbf{u}_{2} \\
\vdots \\
\mathbf{u}_{k}
\end{array}\right]_{k \times M}
$$

Pr o of. According to (3) we have

$$
\hat{\theta}_{k}=B(k) \mathbf{a}_{k}
$$

where

$$
\mathbf{a}_{k} \triangleq U_{k}^{\top} R_{k}^{-1} \mathbf{x}_{k}=U_{k}^{\top} \mathbf{x}_{k}
$$

By replacing the $U_{k}$ in (9) we obtain

$$
\mathbf{a}_{k}=\left[\begin{array}{cccc}
u_{1,1} & u_{2,1} & \ldots & u_{k, 1} \\
u_{1,2} & u_{2,2} & \ldots & u_{k, 2} \\
\vdots & \vdots & \ddots & \vdots \\
u_{1, M} & u_{2, M} & \ldots & u_{k, M}
\end{array}\right]\left[\begin{array}{c}
x_{1} \\
x_{2} \\
\vdots \\
x_{k}
\end{array}\right]
$$

Now we decompose $\mathbf{a}_{k}$ as

$$
\mathbf{a}_{k}=\left[\begin{array}{ccc}
u_{1,1} & \cdots & u_{k-1,1} \\
\vdots & \ddots & \vdots \\
u_{1, M} & \cdots & u_{k-1, M}
\end{array}\right]\left[\begin{array}{c}
x_{1} \\
\vdots \\
x_{k-1}
\end{array}\right]+\left[\begin{array}{c}
u_{k, 1} \\
\vdots \\
u_{k, M}
\end{array}\right] x_{k} .
$$

So, we have the following recursive equation for $\mathbf{a}_{k}$

$$
\mathbf{a}_{k}=\mathbf{a}_{k-1}+x_{k} \mathbf{u}_{k}^{\top}
$$

According to (9) we get the following relation for $B^{-1}(k)$

$$
B^{-1}(k)=\left[\begin{array}{ccc}
u_{1,1} & \cdots & u_{k, 1} \\
\vdots & \ddots & \vdots \\
u_{1, M} & \cdots & u_{k, M}
\end{array}\right]\left[\begin{array}{ccc}
u_{1,1} & \cdots & u_{1, M} \\
\vdots & \ddots & \vdots \\
u_{k, 1} & \cdots & u_{k, M}
\end{array}\right] .
$$

Again we can derive a recursive equation for $B^{-1}(k)$ as

$$
B^{-1}(k)=B^{-1}(k-1)+\mathbf{u}_{k}^{\top} \mathbf{u}_{k} .
$$

Now by replacing the (12) in (8) we have

$$
\hat{\theta}_{k}=B(k) \mathbf{a}_{k}=B(k)\left[\mathbf{a}_{k-1}+x_{k} \mathbf{u}_{k}^{\top}\right] .
$$

According to (8) we have

$$
\mathbf{a}_{k-1}=B^{-1}(k-1) \hat{\theta}_{k-1} .
$$

Therefore by using (16) in (15) we get

$$
\begin{aligned}
\hat{\theta}_{k} & =B(k)\left[\mathbf{a}_{k-1}+x_{k} \mathbf{u}_{k}^{\top}\right] \\
& =B(k)\left[B^{-1}(k-1) \hat{\theta}_{k-1}+x_{k} \mathbf{u}_{k}^{\top}\right] .
\end{aligned}
$$

Now if we replace $B^{-1}(k-1)-\mathbf{u}_{k}^{\top} \mathbf{u}_{k}$ with $B^{-1}(k)$ we have

$$
\begin{aligned}
\hat{\theta}_{k} & =B(k)\left[\left(B^{-1}(k)-\boldsymbol{u}_{k}^{\top} \boldsymbol{u}_{k}\right) \hat{\theta}_{k-1}+x_{k} \boldsymbol{u}_{k}^{\top}\right] \\
& =\hat{\theta}_{k-1}+B(k) \boldsymbol{u}_{k}^{\top}\left(x_{k}-\boldsymbol{u}_{k} \hat{\theta}_{k-1}\right)
\end{aligned}
$$

and the proof is complete.

Proposition 2. The given estimator in (5) is an unbiased estimator for unknown vector parameter.

Pro of. See Appendix A.

It is straightforward to show that the estimator (5) has the following covariance matrix

$$
\Sigma_{\tilde{\theta}_{k}}=\mathrm{E}\left\{(\theta-\hat{\theta})^{2}\right\}=B(k)=\left(U_{k}^{\top} R_{k}^{-1} U_{k}\right)^{-1} .
$$

In Fig. 2 an illustrative scheme of our localized recursive estimation algorithm is shown. 
174 A. Rastegarnia - M. A. Tinati - B. Mozaffary - A. Khalili: A LOCALIZED RECURSIVE ESTIMATION ALGORITHM FOR ...

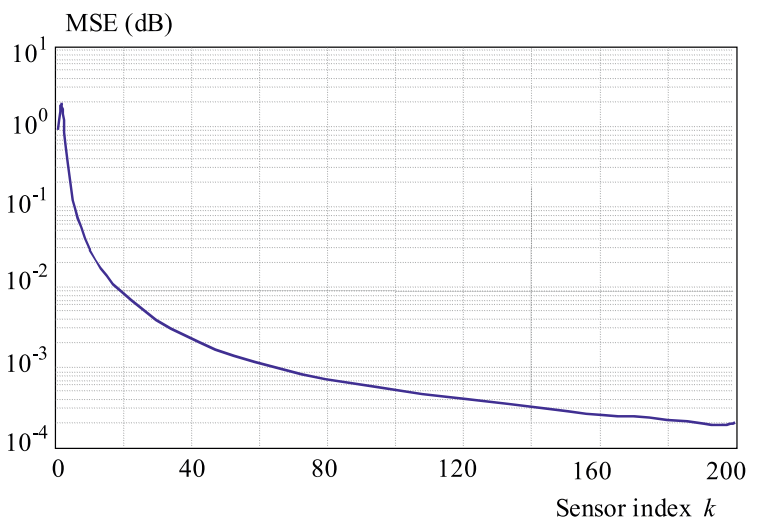

Fig. 3. The MSE performance of the proposed algorithm for $M=2$

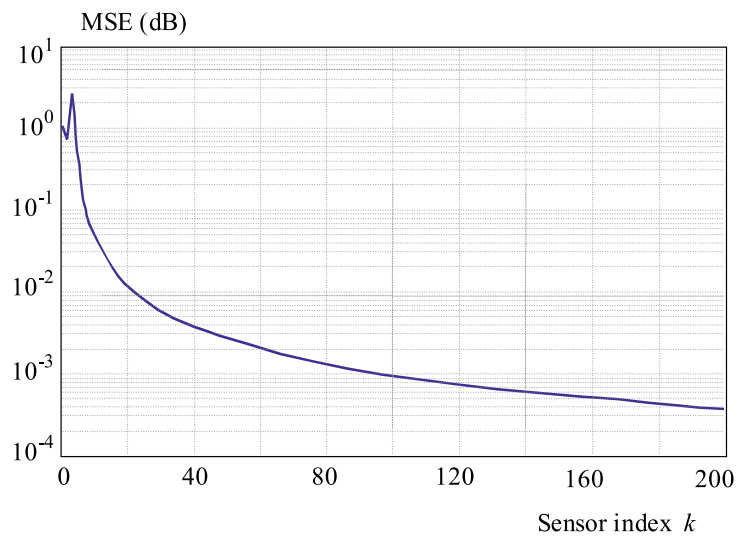

Fig. 5. The MSE performance of the proposed algorithm for $M=4$

\section{NUMERICAL EXAMPLES}

In this section we present the simulation results of the proposed method. To this aim we consider a sensor
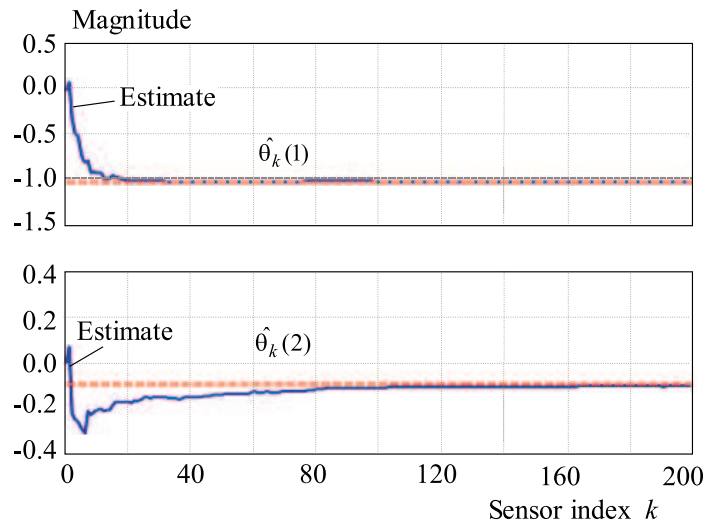

Fig. 4. The tracking performance of the proposed method

network with $N=200$ sensors and assume $\sigma_{k}^{2}=10^{-2}$, $\forall k \in\{1,2, \ldots, N\}$. We also set $\varepsilon=10^{-3}$. The curves are obtained by averaging over 150 runs. To evaluate the performance of the proposed method we use the MSE criteria which is defined as

$$
\mathrm{MSE}=\mathrm{E}\left\{\left(\theta-\hat{\theta}_{k}\right)^{2}\right\} .
$$

In Fig. 3 the MSE performance of the proposed algorithm for $M=2$ (the dimension of the unknown vector) is plotted. As it is clear from Fig. 3, as $k$ increases the quality of local estimate improves. The tracking performance of the proposed method for $\hat{\theta}_{k}(1)$ and $\hat{\theta}_{k}(2)$ is shown in Fig. 4. Here again as $k \rightarrow N$ we have

$$
\lim _{k \rightarrow N} \mathrm{E}\left\{\hat{\theta}_{k}(j)\right\}=\theta, \quad j=1,2, \ldots, M .
$$

In Figs. 5 and 6 the same simulations is done for $M=4$ and same results can be concluded from these
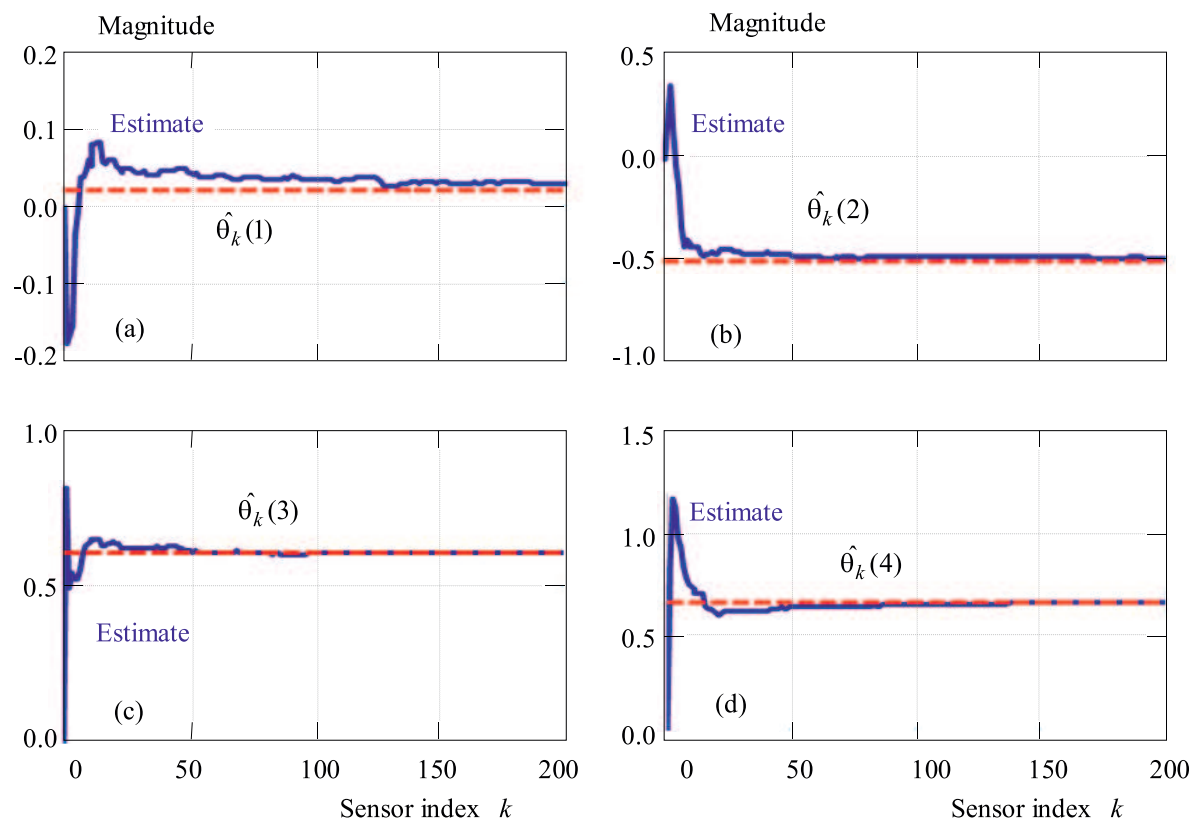

Fig. 6. The tracking performance of the proposed method 


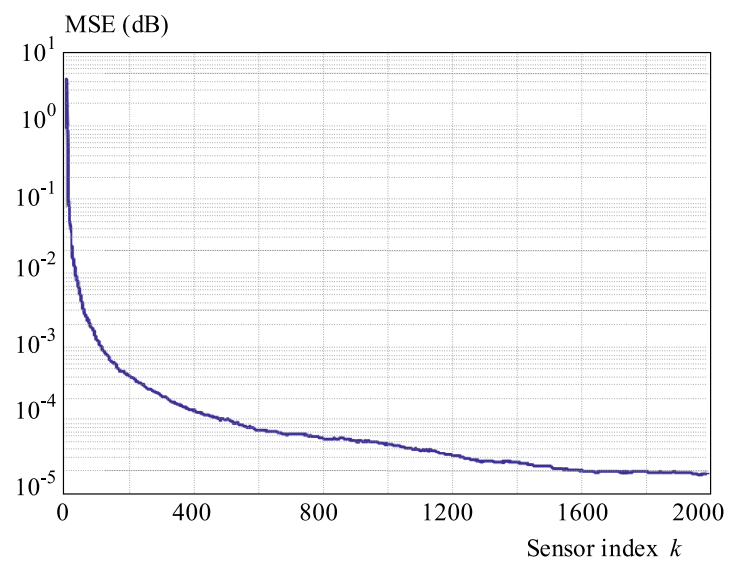

Fig. 7. The MSE performance of the proposed algorithm for $M=4$ and $N=2000$

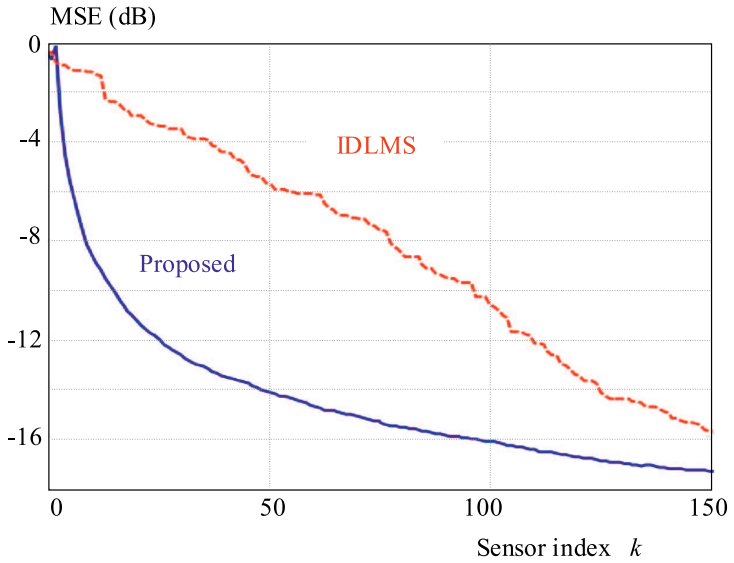

Fig. 8. Comparison of convergence rate between IDLMS and proposed Algorithms

Figs. 5 and 6. In Fig. 7 MSE performance of the proposed algorithm for $M=4$ and $N=2000$ sensors is plotted. It is clear from Fig. 7 that as $N \rightarrow \infty$ the MSE decreases as expected.

In Fig. 8 the convergence rate of the proposed algorithm is compared to the IDLMS algorithm. It must be noted that in IDLMS a closed loop between sensors must be accomplished and in order to get a satisfactory small MSE, it is necessary that the aforementioned loop is done many times. So to compare the IDLMS algorithm with our proposed algorithm we have only considered one loop for IDLMS algorithm. As it is clear from Fig. 8, the proposed algorithm has better convergence rate than the IDLMS.

\section{CONCLUSION}

In this paper a localized recursive estimation scheme for vector parameter estimation in ad hoc wireless sensor networks have been proposed. We considered a linear signal model and assumed that the measurement noises are additive, zero mean, spatially uncorrelated, but otherwise unknown. The classical BLUE can be used to give the desired estimate but due to the practical consideration it can not be perform in wireless sensor networks. To address this problem, in this paper we proposed a localized recursive estimation method which each sensor performs estimation based on its own measurement and the intermediate estimation results from its upstream sensor. After performing estimation, it then transmits its intermediate estimation results to the next downstream sensor. Our mathematical analysis showed that the proposed recursive scheme is an unbiased estimator of the unknown parameter and asymptotically approaches to the estimate that can be achieved by a FC with all real-valued measurements. In addition, the simulation results have been presented to show the performance of the proposed scheme.

\section{APPENDIX PROOF OF THE PROPOSITION 2}

To show this, we use induction argument, $i e$ first show that $\mathrm{E}\left\{\hat{\theta}_{1}\right\}=\theta$ and then assume that it is true for $k$ and show that it is true for $k+1$.

Step I: For $k=1$ we have

$$
\mathrm{E}\left\{\hat{\theta}_{1}\right\}=\mathrm{E}\left\{\hat{\theta}_{0}+B(1) \mathbf{u}_{1}^{\top}\left(x_{1}-\mathbf{u}_{1} \hat{\theta}_{0}\right)\right\}
$$

but, from (1), substituting $x_{1}=\boldsymbol{u}_{1} \theta+v_{1}$. in (20) gives:

$$
\begin{array}{r}
\mathrm{E}\left\{\hat{\theta}_{1}\right\}=\mathrm{E}\left\{\hat{\theta}_{0}\right\}+\mathrm{E}\left\{B(1) \mathbf{u}_{1}^{\top}\left(\mathbf{u}_{1} \theta+v_{1}-\mathbf{u}_{1} \hat{\theta}_{0}\right)\right\}=\mathrm{E}\left\{\hat{\theta}_{0}\right\} \\
\left.+\mathrm{E}\left\{B(1) \mathbf{u}_{1}^{\top} \mathbf{u}_{1} \theta\right\}+\mathrm{E}\left\{B(1) \boldsymbol{u}_{1}^{\top} v_{1}\right\}-\mathrm{E}\left\{B(1) \mathbf{u}_{1}^{\top} \mathbf{u}_{1} \hat{\theta}_{0}\right)\right\} .
\end{array}
$$

Using $\mathrm{E}\left\{v_{1}\right\}=0$ and $B(1)=\left(\boldsymbol{u}_{1}^{\top} \boldsymbol{u}_{1}\right)^{-1}$ in $(21)$ results

$$
\begin{array}{r}
\mathrm{E}\left\{\hat{\theta}_{1}\right\}=\mathrm{E}\left\{\hat{\theta}_{0}\right\}+\underbrace{\mathrm{E}\left\{B(1) \boldsymbol{u}_{1}^{\top} \boldsymbol{u}_{1} \theta\right\}}_{\mathrm{E}\{\theta\}=\theta}+\underbrace{\mathrm{E}\left\{B(1) \boldsymbol{u}_{1}^{\top} v_{1}\right\}}_{0} \\
-\underbrace{\left.\mathrm{E}\left\{B(1) \boldsymbol{u}_{1}^{\top} \boldsymbol{u} \hat{\theta}_{0}\right)\right\}}_{\mathrm{E}\left\{\hat{\theta}_{0}\right\}}
\end{array}
$$

where for the last term in (22) we used $\boldsymbol{u}_{1}^{\top} \mathbf{u}_{1}=B^{-1}(1)$. So for $k=1$ we have $\mathrm{E}\left\{\hat{\theta}_{1}\right\}=\theta$.

Step 2: Now we assume that it is true for any $k$, ie $\mathrm{E}\left\{\hat{\theta}_{k}\right\}=\theta$.

Step 3: According to (5) for $\hat{\theta}_{k+1}$ we have:

$$
\hat{\theta}_{k+1}=\hat{\theta}_{k}+B(k+1) \mathbf{u}_{k+1}^{\top}\left(x_{k+1}-\mathbf{u}_{k+1} \hat{\theta}_{k}\right)
$$

By taking expectation from both sides of (23) we will obtain

$$
\mathrm{E}\left\{\hat{\theta}_{k+1}\right\}=\mathrm{E}\left\{\hat{\theta}_{k}\right\}+\mathrm{E}\left\{B(k+1) \boldsymbol{u}_{k+1}^{\top}\left(x_{k+1}-\boldsymbol{u}_{k+1} \hat{\theta}_{k}\right)\right\}
$$

but the second term in r.h.s of (24) can be written as

$$
\mathrm{E}\left\{B(k+1) \boldsymbol{u}_{k+1}^{\top}\left(\left(\boldsymbol{u}_{k+1} \theta+v_{k+1}\right)-\mathbf{u}_{k+1} \hat{\theta}_{k}\right)\right\} .
$$


Now if we replace (25) in (24) and use the following relations

$$
\begin{aligned}
& \mathrm{E}\left\{\hat{\theta}_{k}\right\}=\theta \\
& \mathrm{E}\left\{B(k+1) \mathbf{u}_{k+1}^{\top} \boldsymbol{u}_{k+1} \theta\right\}=B(k+1) \mathbf{u}_{k+1}^{\top} \mathbf{u}_{k+1} \theta \\
& \mathrm{E}\left\{B(k+1) \mathbf{u}_{k+1}^{\top} v_{k+1}\right\}=0
\end{aligned}
$$

we obtain the desired result

$$
\mathrm{E}\left\{\hat{\theta}_{k+1}\right\}=\theta
$$

\section{Acknowledgment}

The Authors would like to thank Iran telecommunication research center, (ITRC) for their financial support of this research.

\section{REFERENCES}

[1] AKYILDIZ, I.-SU, W.-SANKARASUBRAMANIAM, Y.CAYIRCI, E. : Wireless Sensor Networks: A Survey, Computer Networks 39 (2002), 393-422, Elsevier Publishers.

[2] XIAO, J.-J.-RIBEIRO, A.-LUO, Z.-Q.-GIANNAKIS, G. B. : Distributed Compression-Estimation Using Wireless Sensor Networks, IEEE Signal Process. Mag. 23 (2006), 27-41.

[3] RIBEIRO, A.-GIANNAKIS, G. B.: Bandwidth-Constrained Distributed Estimation for Wireless Sensor Networks, Part I: Gaussian Case, IEEE Trans. Signal Processing 54 (2006), 1131-1143.

[4] RIBEIRO, A.-GIANNAKIS, G. B.: Bandwidth-Constrained Distributed Estimation for Wireless Sensor Networks, Part II: Unknown pdf, IEEE Trans. Signal Processing 54 (2006), 1131-1143.

[5] LUO, Z.-Q.: Universal Decentralized Estimation in a Bandwidth Constrained Sensor Network, IEEE Trans. on Information Theory 51 (2005), 2210-2219.

[6] XIAO, J.-J.-LUO, Z.-Q. : Decentralized Estimation in an Inhomogeneous Sensing Environment, IEEE Trans. on Information Theory 51 (2005), 3564-3575.

[7] LI, H.-FANG, J.: Distributed Adaptive Quantization and Estimation for Wireless Sensor Networks, IEEE Signal Process. Letters 14 (2007), 669-672.

[8] WANG, B.-CHUA, K. C.-SRINIVASAN, V. : Localized Recursive Estimation in Energy Constrained Wireless Sensor Networks, Journal of networks 1 No. 1 (2006), 18-26.

[9] POTTIE-KAISER, W. : Wireless Integrated Network Sensors, Communications of the ACM 43 (2000), 51-58.

[10] SCHIZAS, D.-RIBERION, A.—GIANNAKIS, G. B. : Consensus in ad hoc WSNs with Noisy Links - part I: Distributed Estimation of Deterministic Signals, IEEE Trans. on Signal Process. 56 (2008), 350-364.

[11] LOPES, C.-SAYED, A. H. : Distributed Adaptive Incremental Strategies: Formulation and Performance Analysis, Proc. ICASSP'06, vol. 3, Toulouse, France, 2006, pp. 584-587.

[12] SAYED, A. H.-LOPES, C.: Distributed Recursive LeastSquares Strategies over Adaptive Networks, Proc. 40th Asilomar
Conference on Signals, Systems and Computers, Pacific Grove, CA, 2006, pp. 233-237.

[13] LOPES, C. G.-SAYED, A. H. : Incremental Adaptive Strategies over Distributed Networks, IEEE IEEE Trans. on Signal Process 55 (2007), 4064-4077.

[14] LOPES, C. G.-SAYED, A. H.: Diffusion least-mean-Squares over Adaptive Networks, Proc. ICASSP'07, vol. 3, Honolulu, Hawaii, 2007, pp. 917-920.

[15] CATTIVELli, F.-LOPES, C. G.-SAYED, A. H. : A Diffusion RLS Scheme for Distributed Estimation over Adaptive Networks, Proc. IEEE Workshop on Signal Processing Advances in Wireless Communications (SPAWC), Helsinki, Finland, 2007, pp. 1-5.

[16] LOPES, C. G.-SAYED, A. H.: Steady-State Performance of Adaptive Diffusion Least-Mean Squares, Proc. IEEE Workshop on Statistical Signal Processing (SSP), Madison, W, 2007, pp. $136-140$.

[17] CATtivelli, F.-LOPES, C. G.-SAYED, A. H. : Diffusion Recursive Least-Squares for Distributed Estimation over Adaptive Networks, IEEE IEEE Trans. on Signal Process 56 (2008), $1865-1877$.

[18] WANG, B.-CHAU, K. C.-SRINIVASAN, V.-WANG, W. : Localized Recursive Estimation in Wireless Sensor Networks, Mobile Ad-hoc and Sensor Networks, LNCS, Springer Berlin 3794 (2005), 390-399.

19] KAY, M.: Fundamentals of Statistical Signal Processing: Estimation Theory, Englewood Cliffs, NJ: Prentice-Hall, 1993.

Received 28 August 2009

Amir Rastegarnia received the BS degree and the MS degree in electrical engineering from the University of Tabriz, Iran, in 2004 and 2006, respectively, where he is currently pursuing the $\mathrm{PhD}$ degree in electrical engineering. His current research interests are distributed adaptive estimation, as well as signal processing for communications.

Mohammad Ali Tinati received his BS degree (with high honor) in 1977, his MS degree in 1978 from Northeastern University, Boston, Mass, USA, and his PhD degree from Adelaide University, Australia, in 1999. He served as an academic member of the Faculty of Electrical Engineering since 1979. His main research interests are biomedical signal processing and speech and image processing.

Behzad Mozaffari received his BS degree in 1993 from University of Tabriz and his MS degree in 1996 from K. N. Toosi University of Technology. He is currently a $\mathrm{PhD}$ candidate in electrical communication engineering in the University of Tabriz. His research interests are signal processing and speech processing.

Azam Khalili received the BS degree from K. N. Toosi university of technology, Tehran Iran, and the MS degree from the University of Tabriz, Iran, in 2005 and 2007, respectively, where she is currently pursuing the $\mathrm{PhD}$ degree in electrical engineering. Her current research interests are statistical signal processing, distributed adaptive estimation, as well as speech processing. 